\title{
A New Laparoscope Manipulator with an Optical Zoom
}

\author{
Etsuko KOBAYASHI, Ken MASAMUN, Takeyoshi DOHI, Daijo HASHIMOTO* \\ Department of Precision Machinery Engineering, Graduates School \& Faculty of \\ Engineering, The University of Tokyo, \\ 7-3-1, Hongo, Bunkyo-ku, Tokyo 113, Japan \\ e-mail: etsuko@miki.pe.u-tokyo.ac.jp \\ *Tokyo Metropolitan Police Hospital
}

\begin{abstract}
This report describes a new type of laparoscope manipulator with an optical zoom. The manipulator was designed with regard to its safety in medical settings. It is based on a five-bar linkage mechanism. Back and forth movements of a laparoscope are achieved by an optical zoom. By using the five-bar linkage mechanism with the optical zoom, the manipulator need never interfere with the surgeon or the patient. However, the problem of the quality of the image created by the zoom lens remained. To address this problem, we used a short laparoscope instead of an ordinary laparoscope. In this way, the manipulator achieved a high quality laparoscope image with a sufficient degree of safety
\end{abstract}

\section{Introduction}

Laparoscopic surgery is becoming more popular as a form of minimally invasive surgery [1]. Internal anatomy is observed through the laparoscope and procedures are performed using long forceps that are inserted through trocar sleeves. Compared with open surgery, laparoscopic surgery is more difficult because the surgeon is forced to operate within the limited angle of laparoscopic view and the limited movement of the instruments.

One of the problems in laparoscopic surgery is the fluid manipulation of the laparoscope. Because the surgeon usually uses both hands to manipulate long instruments, it is difficult for the assistant (camera operator) to hold the laparoscope steady and keep the view upright while quickly aiming the scope at the point required by the surgeon.

Several engineers have therefore developed laparoscope manipulators and operator-machine interfaces [2][3][4][5]. The representative manipulator is AESOP (Computer Motion Inc., U.S.A), which has successfully acquired FDA approval as a robot for clinical use. However, laparoscope manipulators developed to date appear to lack adequate safety measures and capacity for sterilization. 
Robots for medical use differ from industrial robots in the following four ways [6].

1. These robots contact the human body directly.

2. They are not allowed repeated trial movements.

3. They are designed to operate easily, because the surgeon is not a specialist in operating robots.

4. They have different functions for each medical procedure.

From the above, we concluded that significant improvements in robot design were needed to satisfy the requirements of the operating theater. The close contact between the robot and the patient requires that safety measures be built into both software and hardware. It is preferable for the robot to be mechanically safe and simple with limited range of movement, rather than to have its safety and usefulness achieved only through the use of complicated software.

Therefore, we have developed a laparoscopic manipulator using a five-bar linkage mechanism and optical zooming [7]. This mechanism allows the manipulator to achieve sufficient safety, and allows for sterilization and minimum obstruction. These are the most important factors for medical robots. However, the problem of image quality achieved by the zoom lens remains.

In this paper, we describe the new type of laparoscope manipulator together with an innovative zooming laparoscope.

\section{Mechanism of the laparoscope manipulator}

We developed the manipulator using a five-bar linkage mechanism (Fig. 1). In this setup, the laparoscope is attached to a holder with two degrees of angular freedom near the insertion site of the abdomen. The manipulator holds the camera end. The manipulator has two degrees of freedom. Its movement in the X-Y plane changes the angle of the laparoscope. A zoom lens substitutes for the back and forth movement of the laparoscope.

To realize the X-Y plane movement of the manipulator, we chose a five-bar linkage mechanism. In this mechanism, the top (P) moves within two degrees of freedom in the $\mathrm{X}-\mathrm{Y}$ plane by changing the angles of two other links (A and $\mathrm{B}$ ). Furthermore, we add another link $(\mathrm{H})$ to the top of the manipulator and a parallel link mechanism (links B, D, E and F). Link (H) remains horizontal at any position by the parallel link mechanism. By link $(\mathrm{H})$ changing in length and setting direction, the surgeon can set the holding point (camera end) at the required position before the manipulation, with the manipulator positioned some distance from the insertion point. 


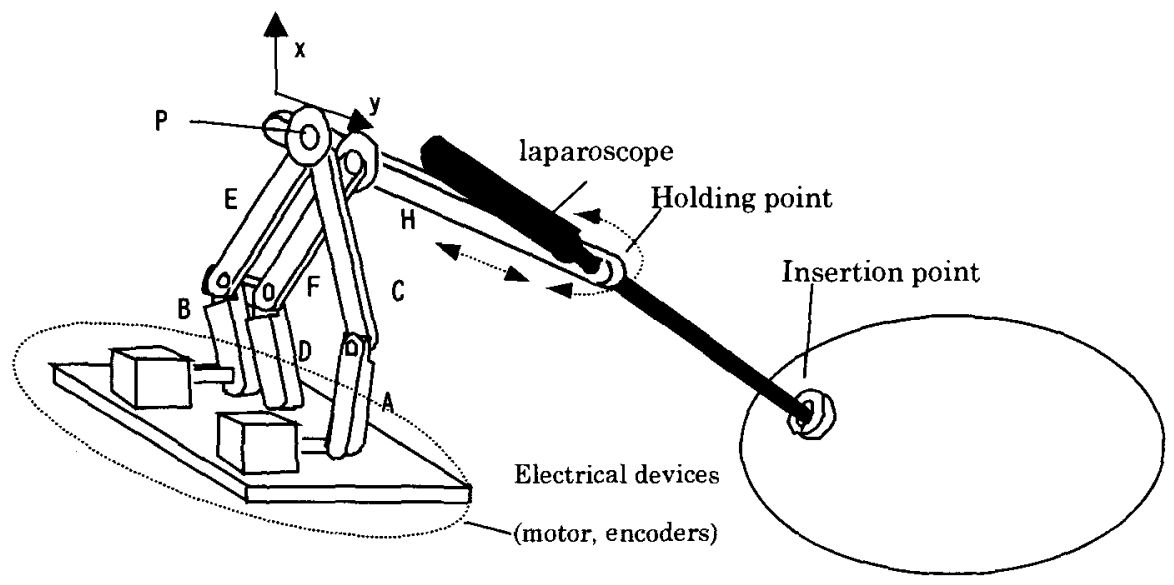

Fig. 1. Mechanism of the manipulator. Because links B, D, E and F make a parallel link mechanism, link $H$ remains horizontal. In this mechanism, all electrical devices can be positioned below.

The advantages of this mechanism are as follows.

1. The manipulator can be placed some distance from the patient's abdomen. As a result the manipulator imposes minimum obstruction on the work of the surgeon and there is little chance of the manipulator coming into contact with the surgeon or the patient.

2. The five-bar linkage mechanism means that the range of movement is limited mechanically. This avoids unexpected movements if the software does not produce accurate responses.

3. A zoom lens substitutes for the back and forth movement of the laparoscope. This minimizes the chance of interference with the organs being examined.

4. Electrical devices such as motors can be positioned below, so that the upper part consists only of simple links. With this mechanism, the upper link is easily removed from the controller, allowing the controller device to be covered with a drape and the linkage part to be sterilized. That is, most parts of the manipulator can be sterilized and only the electrical devices escape sterilization.

We used stepping motors as actuators of our manipulator. The actuators are controlled by a personal computer (PC-9821Xt16, CPU Pentium $166 \mathrm{MHz}$ ) with an open-loop design. To avoid the accumulation of error, the angle of the links is measured by the absolute rotary encoders before use as a control. Encoders measure absolutely the angle of the bottom links at all times, and the initial position does not need to be reset after the surgeon moves the manipulator manually or stops it in case of emergency. The size of this manipulator is $300 \times 300 \mathrm{~mm}$ and the height is a maximum of $400 \mathrm{~mm}$. 


\section{The zooming laparoscope}

\subsection{New design of the zoom lens}

We used an optical zoom instead of the mechanical back and forth movements of a laparoscope. Since the manipulator does not move back and forth, there are two advantages for the laparoscope manipulator. (1) There is little chance of interference with the patient's organs. (2) Because the manipulator needs only two degrees of freedom, its mechanism becomes simple. Therefore, the optical zoom is suited to the laparoscope manipulator.

Requirements for the zoom lens involve its size and the quality of the image. The size should be small so that the surgeon's work is not obstructed. The quality of the image is unavoidably reduced by a zoom lens due to the relay lenses inside the laparoscope and the zoom lens.

To address this problem, we used a short laparoscope instead of an ordinary laparoscope. Fig. 2 shows the construction of our zooming system and Fig.3 shows its picture. We call it a zooming laparoscope. Our zooming laparoscope system consists of a trocar sleeve with optical fibers for a light guide, a short laparoscope and a zoom lens. There are optical fibers in the trocar sleeve to allow light to pass through it. The short laparoscope is the same length as the trocar sleeve and has only one lens at the tip of it. The length of the short laparoscope is $155 \mathrm{~mm}$ and the angle of the field of vision is 80 degrees. The short laparoscope is inserted into the trocar sleeve. For the zoom lens we used two lenses. One is a diameter of $22 \mathrm{~mm}$ and the other is $7 \mathrm{~mm}$. By using a cam mechanism to move the lens, we can change its magnification by turning the outer tube. The second tube is used to focus. The long focal depth means that once we have focused appropriately we do not have to focus again when at another angle. Table 1 shows the specifications of the zooming laparoscope.

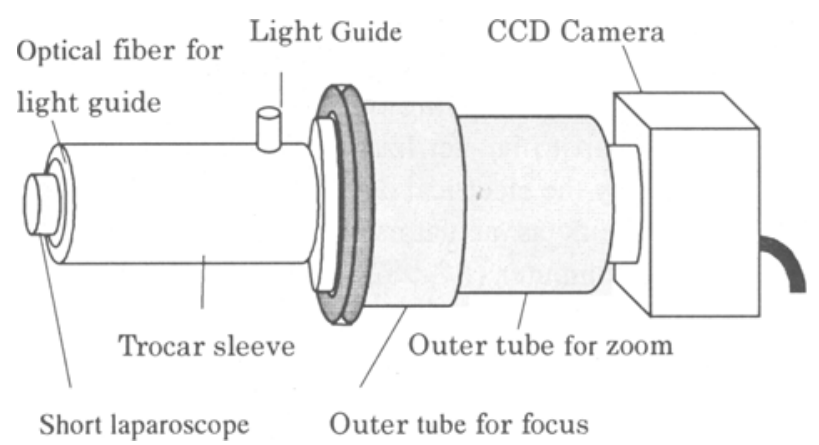

Fig. 2. The construction of a zooming laparoscope 


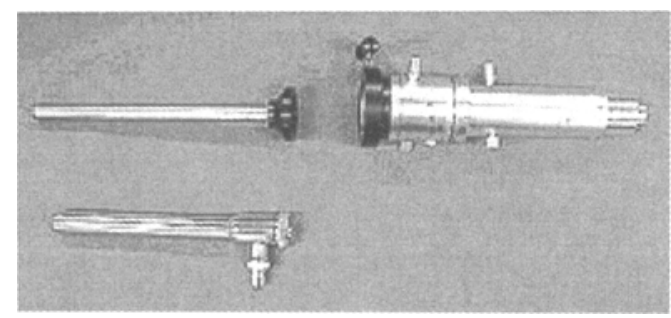

Fig. 3. Zooming laparoscope; left up is a short laparoscope. Left down is a trocar sleeve with optical fibers. Right is a zoom lens.

Table 1. Specifications of the zooming laparoscope.

\begin{tabular}{|c|c|}
\hline CCD camera & SK-1057 3CCD (SINKOKOKI) \\
\hline Magnification & $6.0 \mathrm{x}$ \\
\hline Focal depth $(0.3 x)$ & $5.61(\mathrm{~mm})$ \\
\hline Size: $\quad$ Zoom lens & $\phi 30 \times 158(\mathrm{~mm})$ \\
\hline Trocar sleeve & $\phi 10 \times 155(\mathrm{~mm})$ \\
\hline Short laparoscope & $\phi 14 \times 135(\mathrm{~mm})$ \\
\hline Weight & $800(\mathrm{~g})$ \\
\hline
\end{tabular}

Merits of the zooming laparoscope are as follows.

1. An ordinary laparoscope contains many lenses. On the other hand, the short laparoscope has only one lens. Therefore, by simplifying the optical system, the quality of the image is vastly improved.

2. Because the short laparoscope never comes close to the organs and its length is the same as the trocar sleeve, it never interferes with forceps movements or with the organs being examined.

3. Because the short laparoscope never closes to the organs, it avoids attaching blood to the tip of the laparoscope.

Therefore, we can realize a laparoscope manipulator with a high quality laparoscope image and a sufficient degree of safety by the zooming laparoscope.

Three manual trials of the zooming laparoscope are done on ordinary cholecystectomy. We confirmed that the zooming laparoscope had sufficient magnification an image quality. Fig.4 shows a comparison of laparoscope image between the zooming laparoscope and an ordinary laparoscope. Left is an image of the zooming laparoscope. The surgeon can see the cystic duct in the same magnification with the ordinary laparoscope. 

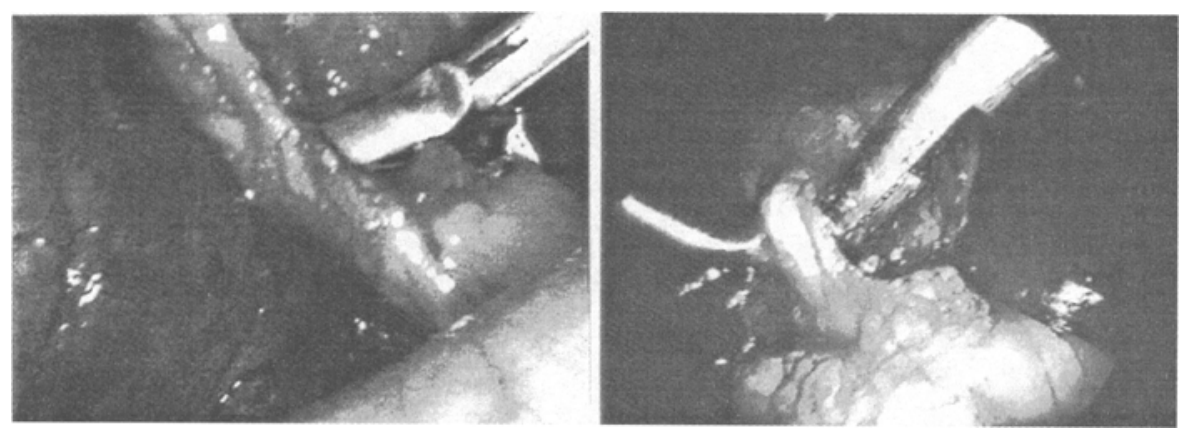

Fig. 4. Laparoscopic images of the cholecystectomy; left is the image of a zooming laparoscope, right is an ordinary laparoscope.

\subsection{Automatic control of a zooming laparoscope}

We developed an automatic control system for the zoom lens (see Fig.5). We used a DC motor (MINIMO co. Itd. 1016 M006G with Gear head 256:1) and a friction belt. The diameter of it is $10 \mathrm{~mm}$. By turning the friction belt, the outer tube of the zoom lens is turned. The value of this design is its simplicity. There are no complicated mechanical parts such as ball bearings. Therefore, with the motor sealed, the whole mechanism can be sterilized.

Fig. 6 shows a laparoscope manipulator with a zooming laparoscope.

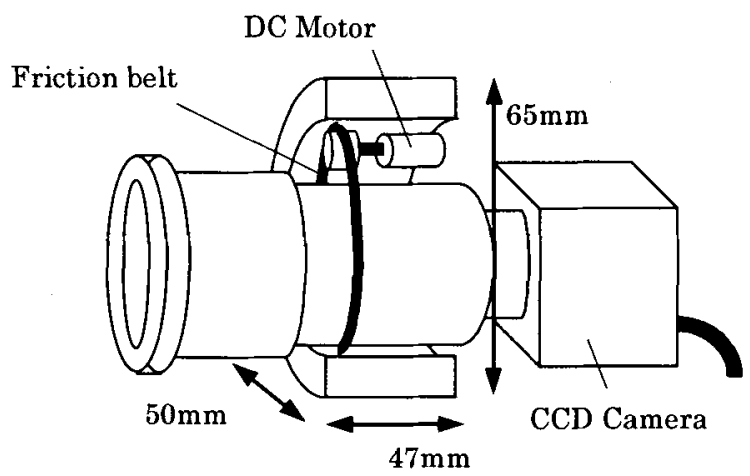

Fig. 5. Automatic control of the zooming laparoscope 


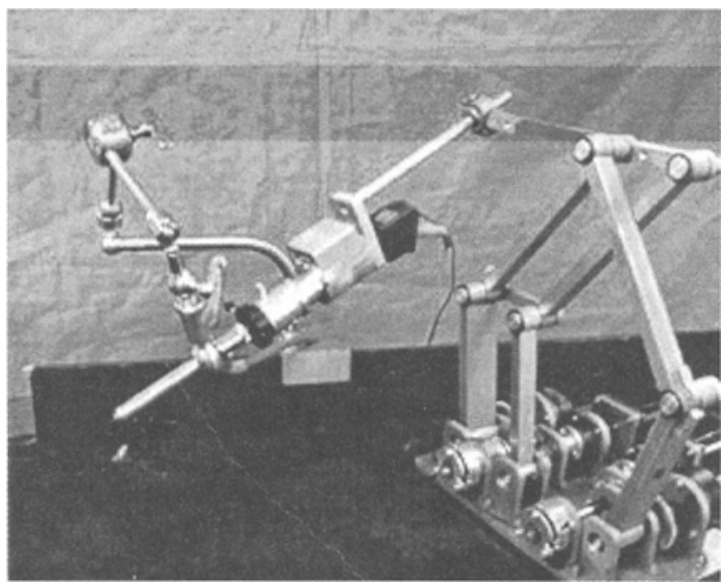

Fig. 6. Laparoscope manipulator with a zooming laparoscope.

\section{Discussion}

We used a five-bar linkage mechanism for the manipulator. This design provided mechanical safety, minimum obstruction to the work of the surgeon and the capacity for sterilization. Furthermore, by putting another link on top of the five-bar linkage mechanism, the surgeon can set the holding point of the manipulator to optimize the range of movement of the manipulator for each operation.

We developed a new type of laparoscope with a zoom lens. By simplifying the optical system, we were able to achieve a high quality image.

A trocar sleeve containing optical fibers is useful as a light source. When placed in other insertion points, light can be obtained from different directions. It has a lot of possibility. For example, light from the different direction makes the shade and then the surgeon can recognize the distance from a forceps to organs.

\section{Conclusion}

We have developed a new type of laparoscope with an optical zoom. Coupled with this zooming laparoscope, a manipulator using a five-bar linkage mechanism achieved mechanical safety and high image quality. We are confident that this manipulator meets the essential requirements for medical use.

This study was partly supported by the Research for the Future Program (JSPS-RFTF 96P00801). 


\section{REFERENCES}

1. Hashimoto D, Hata N, Dohi T: Highly Qualified Laparoscopic Surgery: Gasless 3D operation and intraoperative graphics navigation. Proc. of Computer Assisted Radiology: 811-816 (1995).

2. Taylor RH, Funda J, Eldridge B, Gomory S, Gruben K, LaRose D, Talamini M, Kavossi L, Anderson J A: Telerobotic Assistant for Laparoscopic Surgery. Computer Integrated Surgery: 581-592 (1995).

3. Sackier JM, Wang Y: Robotically Assisted Laparoscopic Surgery: From Concept to Development. Computer Integrated Surgery: 577-580 (1995).

4. Finlay PA, Ornstein MH: Controlling the Movement of a Surgical Laparoscope. IEEE Engineering in Medicine and Biology Vol.14 No.3: 289-299 (1995).

5. Koseki Y, Masamune K, Kataoka H, Masutani Y, Suzuki M, Dohi T, Hashimoto D: Development of an Endoscope Manipulator System for Laparoscopic Surgery. Proc. of Computer Assisted Radiology: 1049 (1996).Dohi T, Hata N, Miyata K, Hashimoto D, Takakura K, Chinzei K,

6. Yamauchi Y: Robotics in Computer Aided Surgery. Journal of Computer Aided Surgery Vol.1 No.1: 4-10 (1995).

7. Kobayashi E, Masamune K, Suzuki M, Dohi T and Hashimoto D: Development of a laparoscope manipulator using five-bar linkage mechanism. Proceedings of Computer Assisted Radiology and Surgery: 825-830 (1997). 